\title{
Discours publicitaire en analyse comparative: des spécificités de genre et des spécificités culturelles, l'exemple de deux spots de publicité pour des marques de café ; « Carte noire » et « Bin Alameed »
}

\author{
Batoul Al-Muhaissen, Romuald Cogné \\ Département de Langues Modernes, Université de Yarmouk, Jordanie
}

Received on: 17-2-2019

Accepted on: 14-11-2019

\section{Résumé}

Ce travail se donne pour but de comparer les communications publicitaires faites par deux marques autour de produits similaires (le café) dans deux communautés sociolinguistiques différentes (en France et en Jordanie). La publicité a ses caractéristiques propres, liées, notamment à ses visées et à la configuration énonciative dans laquelle ce discours s'insère, caractéristiques qui, dans les cas que nous avons choisis, transcendent les communautés susnommées et constitueraient un socle général de spécificités universelles propres à ce genre discursif. Une telle uniformité pourrait également découler du phénomène de mondialisation qui englobe ce discours et les médias qui le relaient.

Nous verrons par ailleurs que si les stratégies discursives déployées sont comparables, les effets sur les représentations de la marque et du produit varient et ce en adéquation avec les normes de représentations socioculturelles partagées entre les individus d'une même communauté linguistique (mais qui divergent entre les deux communautés qui nous intéressent). Un ethos de la marque se construit alors selon ces spécificités culturelles pour activer un réseau de représentations chez le récepteur-consommateur-potentiel afin de créer un sentiment de familiarité qui traduit un «effet de connivence» adapté au public constitué de consommateurs-potentiels de la communauté linguistique (speech community) ciblée.

Mots-clés: scénographie énonciative, analyse du discours, discours publicitaires, représentations socioculturelles, ethos.

\section{Discourse of Advertising: Between Genre and Cultural Specificities; "Carte noire" and "Bin Alameed"}

\begin{abstract}
The present paper aims to compare advertising communications made by two brands about similar products (coffee) in two different sociolinguistic communities (France and Jordan). Advertising as an act has its own specific characteristics that are shaped and chosen based on its goals. The characteristics that are used in our investigation of the advertising communication are universal characteristics that belong to the discourse of this genre. The universal characteristic of the ads discourse or genre is a result of the globalization phenomenon. The paper investigates whether the discursive strategies used by the companies under study are comparable, and whether the effects on the representations of the brand and the product vary and are in line with the norms of socio-cultural representations shared between the individuals of the same linguistic community (but which diverge between the two communities we are interested in). An ethos of the brand is then built according to these cultural specificities to activate a network
\end{abstract}

${ }^{\odot} 2020$ JJMLL Publishers/Yarmouk University. All Rights Reserved. 
of representations in the receiver-potential-consumer in order to create a feeling of familiarity which translates a "connivance effect" adapted to the public consisting of potential consumers of the targeted speech community.

Keywords: enunciative linguistics, discourse analysis, advertising, sociocultural representations, ethos.

\section{Introduction}

La publicité fait partie de notre quotidien, nous la rencontrons sous plusieurs formes: affiches publicitaires, annonces radiophoniques, spots vidéo (télévisés ou sur internet). C'est également un discours que l'on consomme malgré soi: sauf exception, les récepteurs de la publicité ne cherchent pas à regarder ces annonces. Avec la mondialisation et la prépondérance de la société de consommation au niveau international, on assiste à une certaine uniformisation du discours publicitaire (Cook 2001, 3-4). L'homogénéisation de celui-ci sur le plan mondial se remarque notamment au niveau des campagnes lancées par les marques multinationales. Que reste-t-il alors, dans cet espace de pratiques globalisées, des spécificités liées aux sociétés linguistiques? Y-a-t-il toujours, dans l'expression publicitaire des caractéristiques propres à la communauté dans laquelle ce discours s'insère ? Ce sont les questions auxquelles nous allons réfléchir tout au long de ce travail.

Cette étude s'inscrit dans une perspective comparative: nous allons décrire le fonctionnement d'un même type de discours dans deux sociétés différentes afin de faire ressortir les points communs et les différences sur le plan sociolinguistique, au niveau de l'ethos communicatif des deux langues dans les sociétés dans lesquelles elles s'insèrent (Kerbrat-Orecchioni 2011a, 336, 2002). A travers cette étude, nous adhérons à la version faible, communément admise en analyse comparative, de l'hypothèse whorfienne (Kerbrat-Orecchioni 2002, 36): les formes linguistiques utilisées sont des marqueurs de la vision du monde et leur observation permet de reconstruire les représentations communes aux sujets parlants d'une communauté linguistique donnée.

Nous n'avons pas ici la prétention de dégager les éléments universaux d'une manière générale, même au niveau de la publicité. Il s'agit d'un article exploratoire, principalement épistémologique, qui propose une piste de réflexion sur l'influence des préconstruits culturels ${ }^{1}$ sur l'élaboration du discours publicitaire dans les deux communautés linguistiques et l'identification des différences entre les réseaux de représentations invoqués et les différents ethè construits, en convoquant certains effets discursifs. Nous essayons d'illustrer les concepts théoriques en les illustrant à l'aide de l'analyse de deux spots publicitaires.

Notre étude se concentre sur deux spots publicitaires, télévisés et diffusés sur la plateforme Youtube, de deux marques de café en France et en Jordanie. Elle constitue une étude qualitative qui représente le point de départ d'une analyse à plus grande échelle afin de construire plus précisément le profil discursif des langues-cultures étudiées. 
Discours publicitaire en analyse comparative: des spécificités de genre et des spécificités culturelles, l'exemple de deux spots de publicité pour des marques de café ; « Carte noire » et «Bin Alameed »

Nous allons donc dans un premier temps définir la publicité en tant que genre discursif pour ensuite analyser les annonces séparément et ensuite comparer les résultats pour essayer de répondre à notre problématique.

\section{Le discours publicitaire}

Le discours publicitaire est situé dans une logique de consumérisme capitaliste et se distingue des autres genres discursifs de par sa visée performative commerciale (l'acte d'achat par le consommateur), par ses visées illocutoires (vanter le produit et créer et maintenir une image positive de la marque) ainsi que par son caractère « intrusif » dans les médias dans lesquels il s'invite. Pour compenser cet aspect envahissant, la publicité doit donc constamment s'adapter et est en effet en quête constante d'innovation. C'est pourquoi elle doit avoir à la fois une visée de captation et une visée de séduction (Garric 1996, 87). Captation, pour attirer l'attention de son public cible, et séduction, afin de faire acheter le produit vanté par le consommateur. Elle emprunte, dans le discours qu'elle construit, des éléments appartenant à la fois aux «différents domaines de l'activité sociale » (Montserrat Lopez 2006, 130) et aux autres discours présents dans la même communauté discursive. Une annonce publicitaire est alors «un espace de croyances sur des identités, des rôles et des pratiques sociales » (Soulages 2013). Elle représente une plateforme au sein de laquelle sont représentés des clichés d'idéaux de valeurs partagées dans une société donnée à un moment donné.

\section{L'organisation du discours publicitaire (en tant que situation de communication)}

En linguistique énonciative, la scène d'énonciation d'un genre discursif fait intervenir trois scènes: la scène englobante, la scène générique et la scénographie (Maingueneau 2014, 125). Dans la scène englobante, le genre discursif auquel appartient la production est déterminé. La scène générique commande des normes aux usagers du discours: une visée ou finalité, on attribue également aux sujets du discours des rôles avec des caractéristiques prototypiques, un lieu du discours, un support, un répertoire plus ou moins restreint d'outils linguistiques exploitables. La scénographie représente l'aménagement que l'énonciateur fait de la situation d'énonciation (Maingueneau 2014, 129). Des scénographies «types » peuvent être employées dans certains genres de discours comme c'est le cas, nous le verrons, du discours publicitaire.

Tout discours est donc soumis à des normes sociolinguistiques qui sous-tendent sa production et qui dépendent de la configuration énonciative dans laquelle il est produit. Ces normes sociolinguistiques varient en fonction du genre de discours et de la communauté linguistique au sein de laquelle il est produit. Nous nous baserons ici sur le dispositif de mise en scène du langage proposé par Charaudeau (1992, 643-44)pour caractériser la configuration énonciative. Il distingue deux niveaux de participation pour toute situation de communication: les partenaires de l'acte de langage et les protagonistes de l'énonciation. Au premier niveau, on retrouve le locuteur-émetteur et l'interlocuteur-récepteur. Ce sont des êtres sociaux et psychologiques qui émettent et reçoivent effectivement les actes de communication. 
Dans le cas du discours publicitaire, le publiciste ou la société marchande est le locuteur-émetteur et toute personne ayant été témoin de l'annonce est interlocuteur-récepteur. A ces partenaires, qui sont associés par un contrat de communication propre à la situation de communication, s'ajoute un autre niveau de sujets: les protagonistes de l'énonciation qui sont des êtres de parole qui se construisent au sein même de l'acte de parole. Le premier est le locuteur-énonciateur, image construite en fonction des intentions de communication du locuteur-émetteur pour arriver à ses fins, le second est l'interlocuteur-destinataire, image construite par le locuteur, sorte de sujet cible idéalisé. Dans le discours publicitaire, le locuteurémetteur correspondrait aux personnages ou narrateur de l'annonce et l'interlocuteur-destinataire comme le profil ciblé par les publicistes avec toutes les caractéristiques socioprofessionnelles qui lui sont attribuées (par exemple une publicité qui cible des adolescents, des salariés, des retraités...).

Le discours publicitaire est un discours scripté : à l'opposé d'un discours spontané, tout agencement qu'il manifeste est le résultat d'une réflexion minutieuse afin de convaincre, séduire, vendre.

\section{Contrat de « semi-dupes » ${ }^{2}$}

Comme nous l'avons vu précédemment, «tout échange communicatif repose [...] sur un contrat tacite variant selon le genre; chaque discours forme une unité de communication associée à des conditions de production et de réception déterminées » (Montserrat Lopez 2006, 134).

Le discours publicitaire évolue dans un «dispositif triangulaire entre une instance "publiciste», une instance « concurrence » [...] et une instance « public » (Charaudeau 2009, 4).Il se construit alors avec, d'une part, une «visée de séduction» pour amener le consommateur-acheteur-potentiel à effectivement acheter le produit et, d'autre part, une visée de singularisation valorisante (Garric 1996, 77) afin de se démarquer de ses concurrents. L'instance publiciste présente le produit vanté comme étant le meilleur moyen de répondre à un besoin du consommateur-acheteur-potentiel, en opposition à celui de la concurrence, présenté comme inférieur. Tout message publicitaire est donc interprété par le récepteurconsommateur-potentiel comme «une argumentation en faveur de l'objet» (Montserrat Lopez 2006, 134) de la publicité et comme une potentielle dévaluation du produit concurrent. L'image du consommateur-acheteur-potentiel (interlocuteur-destinataire) est construite comme ayant un manque qui doit être comblé par le produit. Le consommateur devrait, en même temps, apprécier le caractère ludique de la publicité par sa visée de connivence (Montserrat Lopez 2006, 143).

\section{Trois principales scénographies locutives ${ }^{3}$}

A travers les relations qui ont lieu entre les différentes instances qui partagent la scène énonciative de la publicité, trois orientations scénographiques émergent en fonction du point focal choisi dans une publicité donnée. La première scénographie gravite autour de l'univers du produit, la seconde développe des avatars du consommateur, images d'un consommateur idéalisé qui a trouvé le bonheur grâce au produit vanté et enfin la troisième scénographie façonne et maintient l'ethos de la marque. 
Discours publicitaire en analyse comparative: des spécificités de genre et des spécificités culturelles, l'exemple de deux spots de publicité pour des marques de café ; « Carte noire » et «Bin Alameed »

○ L'univers du produit

Dans cette scénographie, le produit est le centre de l'interaction entre la marque et le consommateur, la réponse à un besoin que le bienfaiteur que constitue la marque offre généreusement comme solution au manque ou au problème auquel le récepteur est confronté. Le discours emploie en général des formes assertives construisant une logique argumentaire basée sur le logos.

○ Les avatars du consommateur

Dans cette scénographie, c'est une image construite du consommateur-acheteur-potentiel qui est à l'honneur dans la publicité. On lui attribue des caractéristiques sociales à travers des récits de vie idéalisés auxquels le produit et la marque sont associés. Ce n'est plus uniquement la valeur du produit que l'on met en avant, mais également «l'appartenance du consommateur moderne à des identités multiples » (Soulages 2013, 10). Des effets d'indentification opèrent ; on pousse le consommateur-acheteur-potentiel à souhaiter devenir cet être idéalisé avec lequel il partage des caractéristiques ou des aspirations.

o L'ethos de la marque

A travers cette scénographie, le publiciste développe l'image de la marque en lui donnant des attributs positifs. L'image de marque se construit entre un ethos à partir de son ethos prédiscursif (qui existe avant son actualisation dans le discours) et un ethos qui est actualisé dans le discours. Comme le slogan, l'ethos de la marque transcende donc la simple annonce publicitaire. Un système de valeurs que la marque défendrait est alors déployé (morales, éthiques, une garantie de la qualité du produit...). Par exemple, la marque de dentifrice Oral B use de discours "d'experts" afin d'appuyer la qualité de son produit, voire son aspect de produit de recherche scientifique, presque un médicament. L'aspect éthique chez cette marque se retrouve dans la validation par un laboratoire ayant une autorité scientifique présentée comme importante dans les spots publicitaires ${ }^{4}$.

\section{Le slogan}

Le slogan «est d'une part un composant textuel de l'ensemble du contexte publicitaire et d'autre part un acte de communication publicitaire autonome» (Garric 1996, 78). En effet il ne se cantonne pas à une seule annonce publicitaire ni à un seul produit de la marque, mais se retrouve dans l'ensemble des campagnes d'une marque donnée. Court, simple à retenir et ludique, il représente la vitrine de la marque, ce que l'on retient d'elle. Conçu pour être autonome (Maingueneau 2014, 140), il prend sa place à la fin du spot publicitaire, au moment de la « révélation de la marque », il est donc fortement associé à son nom et participe à la construction de son ethos en résumant les valeurs ou les caractéristiques que la marque veut faire ressortir d'elle-même. C'est pourquoi «[...] tout choix linguistique à l'intérieur du slogan répond à une intention de qualification valorisante et singularisante » (Garric 1996, 77). 


\section{Corpus sélectionné}

Le corpus analysé est composé de 2 spots publicitaires: un spot publicitaire de la marque Carte Noire diffusé en France et un spot publicitaire de la marque Bin Alameed diffusé en Jordanie. Ces deux annonces datent respectivement de 2018 et 2017. Les deux spots ont des mises en scènes différentes, et c'est pourquoi leurs descriptions ci-dessous peuvent paraître asymétriques. Nous avons fait le choix de présenter les éléments qui nous paraissaient les plus saillants quant aux stratégies déployées.

\subsection{Carte noire: un univers qui joue sur l'esthétique pour séduire}

Ce spot de 12 secondes est visuellement très recherché esthétiquement: on assiste à un enchainement de gros plans de tasse de café, café moulu... Ces gros plans sont entrecoupés de visuels qui jouent sur des tons sombres et mats.

Texte de la publicité:

Voix off féminine:

«Eh ! Connaissez-vous Douceur Intense de Carte Noire ? Un café doux et généreux. Avec tout le caractère d'un grand café. Carte Noire. Les plus belles couleurs du noir. »

Ici l'énonciateur interpelle dès le début le récepteur-consommateur «Eh», «connaissez-vous ». Cette modalité allocutive implique le récepteur dans le discours en lui posant une question sur la connaissance du produit vanté. Cela permet au discours de capter l'attention du récepteur qui, s'il ne connait pas le produit, devrait avoir envie d'en savoir plus. Le produit est ensuite qualifié d'une manière superlative «doux », « généreux », «tout le caractère », «grand café ». Cette qualification superlative présente le produit comme un produit de grande qualité, qui a des traits humains: générosité, caractère. Cette personnification de l'objet café contribue, avec les oxymores «Douceur intense », «les [...] couleurs du noir » à donner au discours une dimension poétique qui renforce le caractère esthétique de l'annonce.

\section{2. « Bin alameed »: le déploiement de valeurs qui jouent sur des effets de nostalgie}

Spot « muet ${ }^{5} »$ en deux parties $(1 \mathrm{mn} 23 \mathrm{sec})$ :

- Première partie:

En fond sonore on entend l'oud (instrument traditionnel arabe) dont on joue lentement. L'image est ternie afin de la faire passer pour une image enregistrée par une vieille caméra. On voit des grains de café non torréfiés dans un sac en toile de jute posé sur un tapis persan sur une table. On voit les mains d'une personne prendre des grains à l'aide d'une petite pelle et les placer dans un ustensile afin de les torréfier sur un réchaud à huile en cuivre. Les grains torréfiés sont ensuite mis dans un moulin à café en bois et moulu au son grinçant du moulin. Un moulin plus fin est ensuite actionné, on comprend que c'est pour moudre la cardamone ${ }^{6}$ qui est ajoutée à la poudre du café déjà moulu. De l'eau est ensuite versée dans une rakwa ${ }^{7}$ en cuivreà laquelle on ajoute une cuiller du mélange café-cardamone. La rakwa est 
Discours publicitaire en analyse comparative: des spécificités de genre et des spécificités culturelles, l'exemple de deux spots de publicité pour des marques de café ; « Carte noire » et « Bin Alameed»

ensuite mise sur le réchaud en cuivre jusqu'à ébullition. Le café est ensuite servi dans une tasse couleur or sur une soucoupe de même couleur à côté d'une petite assiette assortie sur laquelle sont posés des maamouls ${ }^{8}$, le tout sur une nappe brodée blanche.

- Deuxième partie:

Le rythme de l'oud s'accélère et un instrument à percussion et une guitare l'accompagnent maintenant. On aperçoit de dos une personne en peignoir qui ouvre un placard dans lequel on retrouve les ustensiles utilisés précédemment et une boîte de café «Bin alameed » que la personne sort du placard. On voit ensuite une rakwa en acier inoxydable sur un plan de travail en stratifié. La personne dont on ne distingue que la main met deux cuillers du café dans la rakwa pour ensuite y verser de l'eau chaude d'une bouilloire en inox. La rakwa est ensuite posée sur une plaque à induction jusqu'à ébullition. Le café est ensuite servi dans une tasse du même service que précédemment, accompagnée de la même assiette, mais cette fois avec des carrés de chocolats et sur une table en verre à nu. Un smartphone est posé à côté de l'assiette.

Dans le scénario figuratif de la première partie de l'annonce, les éléments en gras évoquent un imaginaire nostalgique lié au passé avec une axiologie positive (la qualité des produits qui précédait la surconsommation et l'obsolescence programmée). Dans la deuxième partie, le geste traditionnel est reproduit dans un environnement plus moderne et c'est une boîte de café «Bin alameed » qui remplace les grains dans le sac en toile de jute. Les deux activités de préparation du café turc sont mises au même niveau, ce qui est renforcé par le contexte extralinguistique de la vidéo: l'oud qui fait le lien entre les deux scènes du spot et le séquençage similaire de la vidéo.

\section{Analyse comparative}

Les deux publicités empruntent les mêmes types de scénographies locutives: des scénographies orientées sur le produit et sur l'ethos de la marque. Mis à part une interpellation dans chacune des annonces, le consommateur est absent de la scène énonciative, non représenté. C'est le produit qui est la star, porté par une marque qui endosse des valeurs qui sont positivement axiologisées. Cependant les types de valeurs actualisées sont sélectionnés en fonction des représentations sociales liées au produit en vigueur dans la société dans laquelle la publicité et l'ethos est développé par la marque auparavant.

Dans le cas de la publicité française, c'est le café en tant que produit de haute qualité qui est présenté par une marque quasiment de luxe qui utilise l'oxymore, une figure de style poétique d'opposition, qui se retrouve deux fois dans le texte. Cette allusion à la poésie procure au discours une aura à la fois esthétique (renforcée par les images graphiquement recherchées que le texte accompagne dans l'annonce) et de prestige que connote la poésie en général. On retrouve tout cela « résumé » dans le slogan de la marque: «belles» pour l'esthétique et «les couleurs du noir» pour l'oxymore poétique. L'opposition avec la concurrence n'est pas explicite et faiblement marquée. 
Loin de vouloir faire référence à une marque de luxe, l'annonce jordanienne nous plonge au cœur du rituel quotidien de la préparation du café turc au Moyen-Orient arabe. Ce sont des valeurs associées à la tradition, à la seule bonne manière de préparer le café (par opposition tacite à une manière qui serait mauvaise comme, le café filtre ou en dosette). Le produit endosse des valeurs d'authenticité, un café véritable, d'antan, à opposer à un faux café, un non-café (par exemple les autres marques de café, ou le

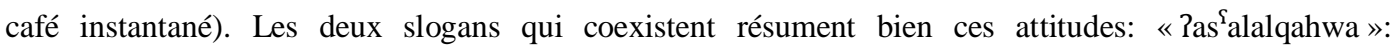
littéralement « l'origine du café » ${ }^{9}$, le mot « Pas $^{\S}$ al » est à rapprocher du mot « as $^{\S}$ irl », « authenticité », le nom de la marque est dénommé comme étant le café originel, ancestral, identique à celui que les générations précédentes utilisaient quotidiennement. Le deuxième slogan confirme l'opposition du produit et de la marque à leurs concurrents: «qarinuhb?ai: bin turi:d » «comparez-le avec le grain que vous voulez $»^{10}$. Ici le consommateur est invité à comparer le café avec n'importe quel autre concurrent. La marque est alors présentée comme indubitablement supérieure aux autres, ne risquant aucune comparaison. Une logique argumentative chargée de pathos (nostalgie) et d'ethos (caractéristiques associées à l'identité discursive du produit) est alors déployée ${ }^{11}$ : ce café est le meilleur car il est identique au café traditionnel, celui que consommaient les générations antérieures.

\section{Conclusion}

La publicité en tant que genre discursif a des spécificités qui lui sont propres à un niveau relativement universel (en tout cas dans la limite très restreinte des cas étudiés). Les deux scénographies énonciatives relevées sont des « hybrides » entre les deux orientations « univers du produit » et « ethos de la marque » qui ont tendance à jouer sur le pathos et l'ethos. Ce sont les qualités du produit qui traduisent des valeurs de la marque («nous ne vendons que le meilleur produit »).

Cependant ces points communs ne sont pas absolus et le discours est contraint par les cadres d'activités sociales partagés par les membres de la communauté discursive qui sont les récepteurs idéalisés par le locuteur-émetteur-publiciste. Les activités qui nous intéressent ici sont la consommation du café et son image dans la société. En effet, nous pensons que les différences observées dans la construction des ethè discursifs de la marque dépendent directement des représentations sociales de l'activité «boire du café ». Le café n'étant pas identifié comme un produit traditionnel du pays, le publiciste choisit de ne pas déployer des valeurs liées à la tradition, l'authenticité comme pour le spot en arabe et présente le produit comme un produit de luxe (le scénario figuratif est comparable à ceux que l'on retrouve dans des publicités pour des parfums ou des bijoux). En se référant au cadre théorique de Maingueneau $^{12}$, on peut dire que les points communs se situeraient au niveau de la scène englobante et les scénographies qui sont employables. Les divergences, quant à elles se retrouvent au niveau de la scène générique qui fournit les normes aux usagers du discours; le répertoire sociolinguistique et culturel dans lequel on pioche pour produire le discours n'est pas le même et le discours produit n'est interprétable qu'à travers ce prisme bien spécifique.

Ce travail représente une étude exploratoire du genre discursif en analyse comparative et ne constitue pas une recherche exhaustive. Nous avons seulement mis en évidence la différence qu'il y a entre 
Discours publicitaire en analyse comparative: des spécificités de genre et des spécificités culturelles, l'exemple de deux spots de publicité pour des marques de café ; « Carte noire » et « Bin Alameed »

spécificités liées au genre du discours publicitaire et au type d'objet (café) et les spécificités liées à la société que la publicité cible.

الخصائص النوعية والثقافية للخطاب الدعائي في التحليل المقارن: دراسة حالة للعلامتين التجاريتين

"كارت نوار" و "بن العميد"

بتول المحيسن، رومويالا كونييه

قسم اللغات الحديثة، جامعة اليرموك، الأردن

الملخص

يهدف هذا البحث إلى مقارنة عمليات التواصل الإعلانية التي تستخدمها علامتان تجاريتان لِمنتِجَ واحد (القهوة) في مجتمعين لغويين جغرافيين مختلفين (فرنسا والأردن). حيث إن للإعلان خصائصه التي ترتبط - على وجه الخصوص - بأهدافه وبالثكل الإعلاني الذي يتجسد فيه الخطاب الدعائي، وهي خصائص تتجاوز - في الحالات التي اخترناها - المجتمعات المذكورة أعلاه، وتشكل أساساً عاماً لخصوصيات الخطاب الدعائي العالمي. ويمكن أن ينتج هذا التشابه أيضاً عن ظاهرة العولمة التي تثمل هذا الخطاب ووسائط الإعلام التي تنقلها. ومن ناحية أخرى، سنرى إن كانت الاستراتيجيات الاستطرادية المستخدمة قابلة للمقارنة، وما إذا كانت التأثيرات على تمثيل العلامة التجارية والمنتج تتنوع، وهذا يتماشى مع معايير التمثيل الاجتماعي والثقافي المشتركة بين الأفراد المنتمين إلى نفس المجتمع اللفوي (ولكنها تختلف بين مجتمعي الدراسة). إذ يتم بعد ذلك بناء أخلاقيات العلامة التجارية وفقاً لهذه الخصائص الثقافيّة من أجل تفعيل شبكة من البيانات التي تمثل المُستقبِلالمستهلكِ المحتمل من أجل خلق شعور بالألفة لديه يُترجم إلى تكيُف المنتج مع الجمهور المكون من مستهلكين محتملين في الأوساط اللفوية المستهدفة. الكلمات المفتاحية: دراسة التركيب المعنوي للمشهد، تحليل الخطاب، الخطاب الدعائي، التصورات الإجتماعية والثقافية،

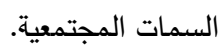




\section{Notes de Fin}

${ }^{1}$ Tel que conçus dans (Montserrat Lopez 2006, 130), à rapprocher aux « représentations sociales » de (Grize 1998, 119).

${ }^{2}$ Le modèle décrit dans cette partie est celui de (Charaudeau 2009, 4-5)

${ }^{3}$ D' après le travail de Soulages (2013).

${ }^{4}$ Planchenault, Gaëlle. 2014. « Mise en scène du discours d'autorité des experts dans la publicité. De l'éloquence du scientifique à la maladresse du praticien. » Mots. Les langages du politique [en ligne], no 105: 105-26.

${ }^{5}$ Il n'y a pas de texte, sauf le slogan à la fin.

${ }^{6}$ Ingrédient essentiel du café turc au Moyen-Orient arabe.

${ }^{7}$ Cafetière servant à faire le café turc.

${ }^{8}$ Pâtisserie fourrée à la pâte de date.

${ }^{9}$ Traduction personnelle des auteurs.

${ }^{10}$ Traduction personnelle des auteurs, ici nous avons pris le parti de mettre l'injonction à la deuxième personne du pluriel alors qu'en arabe le vouvoiement n'existe pas.

${ }^{11}$ Ce qui correspond à une dominance de la scénographie « ethos de la marque ».

${ }^{12} \mathrm{Cf}$. ci-dessus partie 2.

\section{Références}

Charaudeau, Patrick. 1992. Grammaire du sens et de l'expression. Hachette éducation.

Charaudeau, Patrick. 2009. «Le discours de manipulation entre persuasion et influence sociale ». In Acte du colloque de Lyon 2009, consulté le 14 novembre 2019 sur le site de Patrick Charaudeau - Livres, articles, publications. URL: http://www.patrick-charaudeau.com/Le-discours-de-manipulationentre.html

Charaudeau, Patrick, et Maingueneau, Dominique. 2002. Dictionnaire d'analyse du discours. Paris: Editions du Seuil.

Clyne, Michael. 2002. «Contrastive discourse studies ». Cahiers de praxématique, $\mathrm{n}^{\circ}$ 38: 58-84.

Cook, Guy. 2001. The discourse of advertising. London and New-York: Routledge.

Croft, William. 2010. « Relativity, linguistic variation and language universals ». CogniTextes 4.

Demissy-Cazeilles, Olivier. 2007. «Langage et propagande : la traduction française de trois discours de George W. Bush ». Hermès, La Revue 3 (49): 141-48.

Garric, Nathalie. 1996. «Pour une stratégie discursive publicitaire: les adverbes assertifs en -ment». Langage et société, $\mathrm{n}^{\circ}$ 78: 77-88. 
Discours publicitaire en analyse comparative: des spécificités de genre et des spécificités culturelles, l'exemple de deux spots de publicité pour des marques de café ; « Carte noire » et «Bin Alameed »

Grize, Jean-Blaise. 1998. «Logique naturelle, activité de schématisation et concept de représentation ». Cahiers de praxématique, $\mathrm{n}^{\circ} 31: 115-25$.

Kerbrat-Orecchioni, Catherine. 2002. "Système linguistique et ethos communicatif ». Cahiers de praxématique Langue, discours, culture (38): 35-57.

Kerbrat-Orecchioni, Catherine 2011a. Le discours en interaction. Paris: Armand Colin, Collection U Lettres - Linguistique.

Kerbrat-Orecchioni, Catherine. 2011b. «L'illocutoire, ou comment ne pas s'en débarrasser ». In $D u$ système linguistique aux actions langagières, 357-71. Louvain-la-Neuve: De Boeck Supérieur, Collection Champs linguistiques.

Maingueneau, Dominique. 2014. Discours et analyse du discours. Paris: Armand Colin.

M.El-daly, Hosney. 2011. "Towards an Understanding of the Discourse of Advertising: Review of Research with Special Reference to the Egyptian Media. » African Nebula 3: 25-47.

Montserrat Lopez, Diaz. 2006. «L’hétérogénéité du discours publicitaire ». Langage et société 2 (116): 129-45. https://doi.org/10.3917/1s.116.0129.

Paltridge, Brian. 2006. Discourse analysis. An Introduction. London / New York: Continuum.

Planchenault, Gaëlle. 2014. «Mise en scène du discours d'autorité des experts dans la publicité. De l'éloquence du scientifique à la maladresse du praticien. » Mots. Les langages du politique [en ligne], $\mathrm{n}^{\mathrm{o}}$ 105: 105-26.

Sarfati, Georges-Élia. 1989. Éléments d'analyse du discours. Paris: Armand Colin.

Soulages, Jean-Claude. 2013. «L'odre du discours publicitaire». Semen [En ligne], $\mathrm{n}^{\mathrm{o}} 36$. http://journals.openedition.org/semen/9638.

Steffens de Castro, Maria Helena. 2004. «La mise en scène discursive de la publicité ». Sociétés 1 (83): 51-63.

Traverso, Véronique. 2006. Des échanges ordinaires à Damas: Aspects de l'interaction en arabe, approche comparative et interculturelle. Lyon: Presses Universitaires de Lyon/ Institut français du Proche-Orient.

Wierzbicka, Anna. 1985. «Different cultures, different languages, different speech acts ». Journal of Pragmatics 9: 145-78. 


\section{Annexe: transcription de l'alphabet arabe}

Consonnes

\begin{tabular}{|c|c|c|c|}
\hline Arabe & API & Arabe & API \\
\hline$s$ & $?$ & ض & $\mathrm{B}^{\mathrm{f}}$ \\
\hline ب & $\mathrm{b}$ & $b$ & $\mathrm{t}^{\mathrm{s}}$ \\
\hline ت & $\mathrm{t}$ & ظ & $\partial^{\Upsilon}$ \\
\hline$ث$ & $\theta$ & ظ & $z^{\complement}$ \\
\hline$\dot{ث}$ & $\mathrm{~s}$ & $\varepsilon$ & S \\
\hline ث & $\mathrm{t}$ & $\dot{\varepsilon}$ & 8 \\
\hline ج & $\mathrm{d} 3 / 3$ & ف & $\mathrm{f}$ \\
\hline ج & $\mathrm{g}$ & ق & $q$ \\
\hline ج & $\mathrm{j}$ & ق & $?$ \\
\hline$\tau$ & $\hbar$ & ق & $\mathrm{g}$ \\
\hline$\dot{\tau}$ & $\mathrm{x}$ & ق & $\mathrm{k}$ \\
\hline د & d & ك & $\mathrm{k}$ \\
\hline ذ & ð & ك & $\mathrm{t} \int$ \\
\hline j & $\mathrm{z}$ & $J$ & 1 \\
\hline j & $\mathrm{d}$ & م & $\mathrm{m}$ \\
\hline J & $\mathrm{r}$ & $\dot{ن}$ & $\mathrm{n}$ \\
\hline J & в & 0 & $\mathrm{~h}$ \\
\hline س & $\mathrm{s}$ & و & $\mathrm{w}$ \\
\hline ش & $\int$ & ي & $\mathrm{j}$ \\
\hline ص & $s^{\S}$ & & \\
\hline
\end{tabular}

Voyelles

\begin{tabular}{|c|c|c|c|}
\hline Arabe & API & Arabe & API \\
\hline 1 & a: / a: & u & a / a \\
\hline 1 & a: & u & æ \\
\hline$ي$ & i: & $\square$ & i \\
\hline$ي$ & e: & $\square$ & e \\
\hline$و$ & u: & u & u \\
\hline$و$ & o: & u & o \\
\hline
\end{tabular}

